\title{
Entanglement and Dynamics of Spin Chains in Periodically Pulsed Magnetic Fields: Accelerator Modes
}

\author{
T. Boness, S. Bose, and T. S. Monteiro \\ Department of Physics and Astronomy, University College London, Gower Street, London WC1E 6BT, United Kingdom
}

(Received 23 February 2006; published 9 May 2006)

\begin{abstract}
We study the dynamics of a single excitation in a Heisenberg spin-chain subjected to a sequence of periodic pulses from an external, parabolic, magnetic field. We show that, for experimentally reasonable parameters, a pair of counterpropagating coherent states is ejected from the center of the chain. We find an illuminating correspondence with the quantum time evolution of the well-known paradigm of quantum chaos, the quantum kicked rotor. From this we can analyze the entanglement production and interpret the ejected coherent states as a manifestation of the so-called "accelerator modes" of a classically chaotic system.
\end{abstract}

DOI: 10.1103/PhysRevLett.96.187201

There is considerable interest in the fidelity of quantum state transmission and entanglement measures in spin chains because of their relevance to quantum-information applications. In [1] state transmission in a Heisenberg chain was investigated. In [2] it was shown that such a chain, in the presence of an external, static, parabolic magnetic field, can give perfect transmission of coherent spin states of appropriate width. Obtaining coherent states of specified widths represents a technical challenge though.

Here, we investigate the dynamics of a Heisenberg spinchain subjected to short, time-periodic pulses from an external parabolic magnetic field. We find that this provides an effective technique for generating well-defined coherent states, starting from a single excitation at the center of the spin chain. The key to the analysis is that we note, for the first time, a close correspondence between the time evolution of a Heisenberg chain and that of the well-known quantum kicked rotor (QKR) [3,4] in its quantumresonance regime [5]. Our additional parabolic external field extends the correspondence between the Heisenberg spin chain to the nonresonant QKR. Both the nonresonant and resonant QKR have been well investigated experimentally with cold atoms in optical lattices [5,6].

There is also much current interest in the interface between quantum chaos and quantum information [7]. In some studies of entanglement measures, quantum chaos is generated by extrinsic disorder [8]; in others, with a clean but chaotic Hamiltonian [9,10]. The question of whether chaos aids or hinders entanglement generation relevant to quantum-information applications has not yet yielded a clear answer [10]. In [11] it was shown that a class of kicked Ising-type chains have quantum behavior related to those of one-body "image" systems with a well-defined classical limit, which can be chaotic or integrable.

However, to our knowledge, the correspondence between the dynamics of the QKR, a leading paradigm of quantum chaos and the Heisenberg chain, a system of such key interest in quantum-information, has not been noted or exploited previously. We show it means that with the
PACS numbers: 75.10.Pq, 03.67.Hk, 03.67.Mn, 05.45.Mt

pulsed parabolic field, we can employ certain "textbook" [12] expressions found for the QKR and the standard map to describe the entanglement properties. It means also that we see not only generic forms of quantum chaotic behavior in the spin chain, like exponential localization [shown in [4] to be analogous to Anderson localization] but also phenomena (such as "accelerator modes") due to additional correlations specific to a "clean" chaotic systemand to the QKR, in particular. We show that, as we can remain in the one-excitation sector with this Hamiltonian, there are new possibilities for quantum-information applications from the entanglement properties of the accelerator modes.

We consider a time-periodic Hamiltonian of the form:

$$
\mathbf{H}=H_{\mathrm{hc}}+\sum_{n=1}^{N} \frac{B_{Q}}{2}\left(n-n_{0}\right)^{2} \sigma_{z}^{n} \sum_{j} \delta\left(t-j T_{0}\right),
$$

where $\quad H_{\mathrm{hc}}=-\frac{J}{2} \sum_{n} \sigma^{n} \cdot \sigma^{n+1}-\sum_{n} B \sigma_{z}^{n} \quad$ is the Hamiltonian for the Heisenberg chain studied in [1]. $T_{0}$ is the period of the pulses; $B_{Q}$ is the amplitude of the applied parabolic magnetic field and $n_{0}$ is the site at which the field is a minimum. $N$ is the length of the chain: here, $N \gtrsim 100$. The spin-transmission properties for the timeindependent part were investigated in [1]: for a nonzero static field, where $\hbar B \gg k T$ one may restrict the study to the single-excitation regime [i.e., restricted to the basis of states $|\mathbf{j}\rangle$, which have a spin-up at a single site $j$ on the chain but all other spins down (along $-\hat{z}$ )].

The eigenstates of $H_{\mathrm{hc}},|\tilde{m}\rangle$ [13] are delocalized along the chain and may be written in the $|\mathbf{j}\rangle$ basis [1]:

$$
|\tilde{m}\rangle=a_{m} \sum_{j=1}^{N} \cos \left[\frac{\pi}{2 N}(m-1)(2 j-1)\right]|\mathbf{j}\rangle,
$$

where $a_{m}=\sqrt{\frac{2-\delta_{m 1}}{N}}$. Using the eigenstates, an analytical form for the time-evolution operator $U_{\mathrm{hc}}(t, 0)=$ $\exp \left\{-\frac{i}{\hbar} H_{\mathrm{hc}} t\right\}$ may be obtained in the single-excitation basis: 


$$
\left\langle\mathbf{r}\left|U_{\mathrm{hc}}(t)\right| \mathbf{s}\right\rangle=\sum_{m=1}^{N} a_{m}^{2} e^{-2 i J t[1-\cos (\pi / N)(m-1)]} \cos \left[\frac{\pi}{2 N}(m-1)(2 r-1)\right] \cos \left[\frac{\pi}{2 N}(m-1)(2 s-1)\right],
$$

where we disregard the uninteresting overall phase due to the uniform static field $B$ (or formally set $2 B T_{0}=2 \pi$ ). For the periodically pulsed system described by the Hamiltonian in (1), the matrix elements of the one-period timeevolution operator $U\left(T_{0}, 0\right)$, in the single-excitation basis, are:

$$
U_{\mathrm{rs}}\left(T_{0}\right)=e^{-i\left(B_{Q} / 2\right)\left(r-n_{0}\right)^{2}} \cdot\left\langle\mathbf{r}\left|U_{\mathrm{hc}}\left(T_{0}\right)\right| \mathbf{s}\right\rangle .
$$

In Fig. 1, we show the effect of repeated application of (4), using the matrix elements in (3), on the state $\psi(t=$ $0)=\left|n_{0}\right\rangle-$ a state initialized on a site at, or very near, the center of the chain. The spin-amplitude spreads out into an irregular distribution peaked around the site $n_{0}$. But most strikingly, we can see a pair of counterpropagating spikes, "hopping" around $2 \pi / B_{Q} \simeq 94$ spin sites each consecutive period. In order to analyze this behavior, we reexamine the form of $U_{\mathrm{rs}}^{\mathrm{hc}}\left(T_{0}, 0\right)$.

It is easy to see that, as $N \rightarrow \infty$, (3) becomes the discretized version of an integral: i.e., $\left\langle r\left|U^{\mathrm{hc}}\right| s\right\rangle \rightarrow F_{\mathrm{rs}}$, where

$$
\begin{aligned}
F_{\mathrm{rs}}= & \frac{e^{-2 i J T_{0}}}{2 \pi} \int_{0}^{\pi}[\cos (r+s-1) x+\cos (r \\
& -s) x] e^{2 i J T_{0} \cos x} d x
\end{aligned}
$$

The $x=\pi(m-1) / N$ is nominally a position coordinate but in fact represents motion through the subspace of $|\tilde{m}\rangle$, the eigenstates of $H_{\mathrm{hc}}$. Equation (5) can be compared with the time-evolution operator of one of the most studied systems in quantum chaos, the QKR [3].

The QKR corresponds to the Hamiltonian $H=$ $\frac{P^{2}}{2}-K \cos x \sum_{n} \delta(t-n)$, where $K$ is the kick strength and $T=1$ is the kicking period. The equations of motion for its classical limit produce the "standard map," the textbook paradigm of classical Hamiltonian chaos [12]. The timeevolution operator of the $\mathrm{QKR}, U^{\mathrm{QKR}}$, is generally given in a plane wave basis $|l\rangle=1 / \sqrt{2 \pi} \exp \{i l x\}$ with $l=$ $0,1,2, \ldots L$, where $L$ is where we truncate our basis. Then, $\left\langle n\left|U^{\mathrm{QKR}}\right| l\right\rangle=e^{-i l^{2} \hbar / 2} i^{n-l} J_{n-l}(K / \hbar)$ where $J$ denotes an ordinary Bessel function. If, e.g., $\hbar=2 M \pi$, with $M=1,2,3 \ldots$ we obtain simply $\left\langle n\left|U^{\mathrm{QKR}}\right| l\right\rangle=$ $\pm i^{n-l} J_{n-l}(K / \hbar)$, i.e., an instance of the quantumresonance regime [also much studied in cold atom experiments [5] ]. In the experiments, $\hbar$ represents an effective value obtained from the optical lattice parameters and kick period $T$, typically of order $\hbar \sim 1[6,14]$.

However, since parity is conserved, the basis states of the QKR are generally symmetrized, so $\psi_{l}^{ \pm}=1 / \sqrt{2}[|l\rangle \pm$ $|-l\rangle]$. It is evident that, to within an overall phase, the form of the integral in (5) is equivalent to the "kick" part of the QKR operator:

$$
F_{\mathrm{rs}} \equiv\left\langle\psi_{r-1 / 2}^{+}\left|e^{i(K / \hbar) \cos x}\right| \psi_{s-1 / 2}^{+}\right\rangle
$$

if $2 J T_{0}=K / \hbar=\beta$. Above, $|r-1 / 2\rangle$ indicates plane waves shifted by half a quantum, i.e., $|r-1 / 2\rangle=$ $\frac{1}{\sqrt{2 \pi}} e^{i(r-1 / 2) x}$. Further,

$$
F_{\mathrm{rs}}=\left\langle\psi_{r-1 / 2}^{+}\left|e^{i(K / \hbar) \cos x}\right| \psi_{s-1 / 2}^{+}\right\rangle \simeq i^{r-s} J_{r-s}(\beta)
$$

provided we neglect terms which are only significant at the edges of the basis (i.e., $r \approx 1$ or $r \approx N$ ). Note that for a spin ring, these "edge" corrections are entirely absent: the time evolution of a Heisenberg spin ring is entirely equivalent to the kick part of the QKR. The addition of the parabolic-field kick completes the analogy with the full QKR: the fact that the parabolic field is now the $\delta$-kick term, while for the QKR the kinetic energy provides the time-independent term, represents only a simple rescaling of the parameters in $U\left(T_{0}, 0\right)$.

Note that all our numerics employ the matrix element (3) and not the QKR form in (7). Nevertheless, we found the spin-chain dynamics are sufficiently analogous that we can simply make the substitutions: (a) $B_{Q}=\hbar$ is an effective value of Planck's constant. (b) $2 J T_{0} B_{Q}=K$, where $K$ is the stochasticity parameter which fully determines the classical dynamics of the kicked rotor; e.g., $K \geq 4.5$ in-

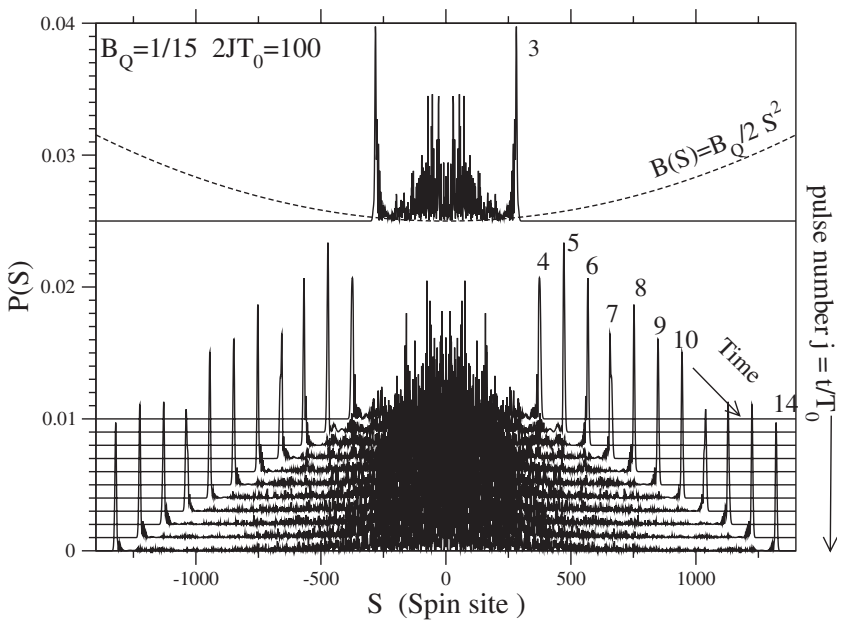

FIG. 1. Time evolution of the state $\psi(t=0)=\left|\mathbf{n}_{\mathbf{0}}\right\rangle$ (i.e., initialized with a single spin-excitation at the center of the chain) showing the effect of accelerator modes. $P(s)$ represents the probability of finding the excitation at site $s$. The accelerator modes are the "spikes" at the leading edge of the distribution. They correspond to a counterpropagating pair of coherent states ejected from the center. We take $B_{Q}=1 / 15$ and $2 J T_{0}=100$. The upper line is at $t=3 T_{0}$; the lower curves correspond to consecutive periods $j T_{0}$ with $j=4,5,6 \ldots$ as numbered. The dotted line indicates the form of the parabolic field (scaled by a constant factor) which is pulsed on/off every period at times $t=$ $j T_{0}$. The accelerator modes represent over $25 \%$ of the total probability; they advance an equal distance (shown below to be $2 \pi / B_{Q} \simeq 94$ spin sites) each period, and after just 3 pulses are well separated from the central, "chaotic" remnant. 
dicates fully chaotic dynamics. We can then directly apply the well-known QKR results to the spin dynamics.

(1) Short-time diffusion of spin excitation. -For $K \gtrsim 4$ the QKR initially displays diffusion of momentum, $\left\langle p^{2}\right\rangle \approx$ $D(K) t$, which follows the classical behavior: the form $D(K) \approx K^{2} / 2\left\{1-2 J_{2}(K)+2 J_{2}(K)^{2}\right\} \quad$ [15] is obtained from a study of the classical diffusion for the standard map. The first term in the expression for $D$ represents uncorrelated diffusion (equivalent to a random walk); the $J_{2}(K)$ terms result from short-time correlations present in the classical kicked rotor. We obtain analogous behavior in our pulsed spin chain; substituting $2 J T_{0} B_{Q}=K_{s}$, and starting out with a spin initialized on a site $s=s_{0}$ anywhere on the chain, we find $\left\langle\left(s-s_{0}\right)^{2} B_{Q}^{2}\right\rangle \approx D\left(K_{s}\right) t$.
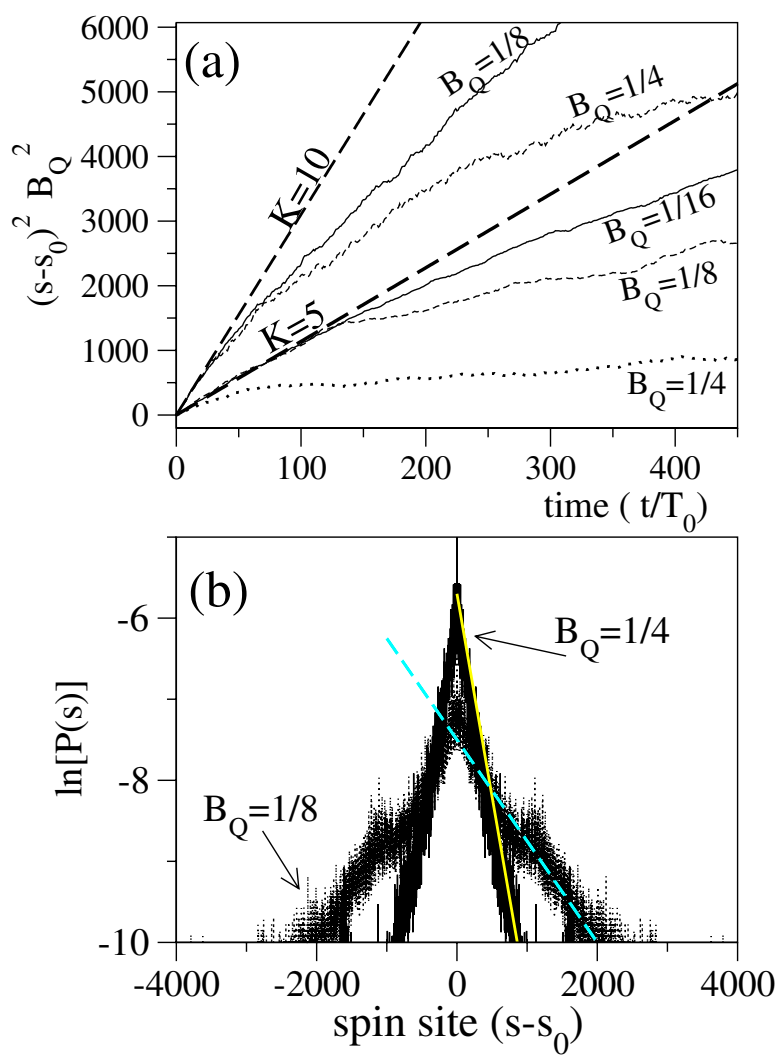

FIG. 2 (color online). (a) Shows that the rate of spreading of a spin excitation initially at an arbitrary site $\left|s_{0}\right\rangle$ in the chain, at short times, is determined solely by the "classical stochasticity parameter" $K_{s}=2 J T_{0} B_{Q}$. At longer times, the spreading saturates. The straight line indicates the behavior expected of the classical chaotic kicked rotor. The smaller the effective value of Planck's constant, $B_{Q}$, the longer the spin spreads linearly at the "classical" rate. Note that in the accelerator mode regime, however, the increase would be quadratic in time, not linear. (b) Shows that at sufficiently long times the spin probability distribution saturates into an exponentially localized form. This is the analogue of Anderson localization in a disordered metal. $P(s) \sim \exp \left\{-2\left|s-s_{0}\right| / L\right\}$ so $\ln [P(s)] \sim 2 / L$ takes the characteristic "triangular form." The straight lines correspond to $L=$ $\frac{K_{s}^{2}}{4 B_{Q}^{2}}$ for $K_{s}=10$ and agree well with the numerically calculated distributions.
Figure 2(a) compares the classical and quantum diffusion rates. For $K_{s}=10$, the correction due to short-range correlations is large, but for $K_{s}=5$, we find $D \approx K_{s}^{2} / 2$ since $J_{2}(5) \approx 0$. After the so-called "break time," $t^{*} \sim$ $\left(K_{s} / B_{Q}\right)^{2}$ the quantum diffusion no longer follows the classical behavior and the spin-excitation stops spreading.

(2) Dynamical localization. - The Floquet states [eigenstates of $\left.U^{\mathrm{QKR}}(T, 0)\right]$ of the QKR are known to be delocalized in the resonant regime; hence, as investigated in [1], a spin excitation can transmit (imperfectly) from one end of the chain to the other. In the nonresonant case, however, the corresponding Floquet states exhibit dynamical localization, a phenomenon analogous to Anderson localization in a disordered metal [4]. Hence, for the spin chain in pulsed parabolic field, a single excitation anywhere in the chain will spread for a finite time and will then "freeze" (localize) at the break time, $t^{*}$. For $t>t^{*}$,

$$
P_{\text {loc }}(s) \sim \frac{1}{L} \exp \left\{-2\left|s-s_{0}\right| / L\right\} .
$$

The quantum localization length $L \simeq\left(2 J T_{0}\right)^{2} / 4$. Figure 2(b) demonstrates the typical probability distribution of a dynamically localized state.

(3) Entanglement. - Our knowledge of the time evolution and spreading of the spin distribution enables us to estimate some entanglement measures for the oneexcitation regime, in particular, the $Q$ measure [16] and the concurrence [17]. The former is a measure of the global entanglement for a pure (multipartite) state:

$$
Q(|\psi\rangle)=\frac{4}{N}\left(1-\sum_{k=1}^{N}\left|\alpha_{k}\right|^{4}\right) \sim \frac{4}{N}\left(1-\frac{1}{2 L}\right),
$$

where the $\alpha_{k}$ are the amplitudes when the state is projected on to the basis of spin sites, $\{|k\rangle\}$. We note the relation of this formula to the well-known inverse participation ratio, $R=1 / \sum\left|a_{k}\right|^{4} ;$ for a localized system $R \simeq 2 L$.

We assign a measure to the bipartite entanglement of two separated sites $i$ and $j$ with the concurrence. Given a pure state in the single-excitation basis $C_{i, j}=2\left|\alpha_{i}\right|\left|\alpha_{j}\right|$. With the exponentially localized form, (8):

$$
C_{i, j} \sim \frac{2}{L} \exp \left\{-\frac{1}{L}\left(\left|i-s_{0}\right|+\left|j-s_{0}\right|\right)\right\}
$$

if the separation between two sites $d_{i, j}=\left|i-s_{0}\right|+\mid j-$ $s_{0} \mid>0$, the maximum of the concurrence occurs at $L \sim d$, with the value $\sim \frac{2}{d} e^{-1}$.

(4) Accelerator modes. -For values of $K \approx 2 \pi$, transporting islands of stability reappear in the classical phase space. Their phase-space area is $\sim 1 / 10$ and hence can support quantum states if $\hbar \leqslant 1 / 10$. Their effect has been experimentally observed for cold cesium atoms in pulsed standing waves of light [18]. Because of the relatively large effective values of $\hbar \gtrsim 1$ in the atomic experiments, their effect there was diluted by the chaotic "sea" of trajectories; nevertheless, the accelerator modes (and 
Levy flights due to their environs) manifested themselves as an enhancement of the diffusion for $K \simeq 2 \pi$.

In the spin chains there is no evident bar to a low $\hbar=$ $B_{Q}$; it simply requires a weaker magnetic field. Accelerator modes, in fact, are stable for a broad parameter range $K=$ $\alpha 2 \pi$ where $\alpha=1.03-1.10$ so there is no need for very precise fine-tuning of the parameter $2 J T_{0}$. The associated islands of stability span a width (of spin sites) of order $\Delta s \sim \frac{1}{10} 2 \pi / \hbar$ (about 10 sites for Fig. 1), so there is no need to prepare the initial state exactly at the center of the chain either, in order for the initial state to overlap strongly with the accelerator islands.

We find useful coherent states are obtained provided $B_{Q} \lesssim 1 / 5$. The width of these states is simply determined by the effective $\hbar$ : we verified numerically that their form is given by $\psi\left(s, t=j T_{0}\right) \simeq A_{j} \exp \left\{-B_{Q}\left(s-s_{j}\right)^{2}\right\}$, where $s_{j}=2 \pi j / \hbar$ and $j$ is the pulse number. We find that, for $j \simeq$ 1 the coherent state pair represents about $30 \%$ of the total probability. For finite $\hbar$ we expect the amplitude to gradually "tunnel" out of the accelerator island: for $\hbar=1 / 10$ we estimated the decay numerically: $\left|A_{j}\right|^{2} \simeq\left|A_{j=1}\right|^{2} \times$ $\exp \{-j / 24\}$, so even after 30 pulses there is a substantial amplitude. For $\hbar=1 / 15$ (shown in Fig. 1) the island can in fact support more than one eigenstate, leading to a slight oscillation in its amplitude. It only requires $2-4$ pulses to cleanly separate the traveling states from the chaotic remnant, so even $\hbar \simeq 1 / 5$ would give coherent states with probability $\geq 20 \%$. These could then be taken into a static, parabolic-field region and transmitted onwards with perfect fidelity [2].

For an actual realization we might suggest $B_{Q} \sim 1 / 10$; in an experiment, $B_{Q}=\tilde{B}_{Q} \delta t$ where $\delta t \ll T_{0}$ is the pulse duration. If our maximum magnetic field $B_{\max }$ is of order 0.1 Tesla, $B_{\max } \sim(N / 2)^{2} \tilde{B}_{Q} \sim 10^{-6}$ a.u., for $N \sim$ 100-1000, implies $\tilde{B}_{Q} \sim 10^{-10}$ a.u. so the pulse duration $\delta t \sim 10^{9}$ a.u. $\sim 25 \mathrm{~ns}$. As we have made a split-operator approximation in Eq. (4), we require $2 J \delta t$ is a small phase, whereas $2 J T_{0}$ is significant. This constrains $2 J \sim 10^{-9} \sim$ 1-10 MHz, so we are in a weak spin-coupling regime, for this choice of parameters.

We now propose an application of the accelerator modes in context of quantum communication. It has been noted that encoding quantum information in Gaussian wave packets of excitation (where the presence and absence of the wave packet depicts logical $|1\rangle$ and $|0\rangle$ states of a qubit) is a useful way of transmitting it down a Heisenberg coupled chain of spins $[2,19]$. However, precisely how to create a superposition of the presence and absence of a Gaussian wave packet remained unclear. The work presented here suggests, in fact, that after 3-4 kicks one creates a superposition of two Gaussian wave packets traveling in opposite directions and a exponentially localized state in the middle of a Heisenberg spin chain. If one measures the exponentially localized part and does not find the spin excitation there (which can have nearly 30\% probability of occurrence for appropriately chosen parame- ters), then the spin chain is projected on to a superposition of oppositely traveling Gaussian wave packets of excitation. If one denotes the left and right traveling wave packets as $\left|G_{L}\right\rangle$ and $\left|G_{R}\right\rangle$ (which are each a Gaussian distribution of spin-up), and $\left|0_{L}\right\rangle$ and $\left|0_{R}\right\rangle$ as the absence of such a wave packet (which are each an all spin-down state at the sites where $\left|G_{L}\right\rangle$ and $\left|G_{R}\right\rangle$ would otherwise be), then the maximally entangled state $\left|G_{L}\right\rangle\left|0_{R}\right\rangle+\left|0_{L}\right\rangle\left|G_{R}\right\rangle$ is effectively created. This state can now be distributed among well separated parties by switching on a constant parabolic field (instead of the kicks) in which a Gaussian wave packet can travel for a significant distance without distortion [2].

In conclusion: we have demonstrated that there is a close correspondence between a spin chain in a pulsed quadratic field and the well-known chaotic kicked rotor; we find potential applications in quantum-information processing.

T. B. acknowledges support from the EPSRC.

[1] S. Bose, Phys. Rev. Lett. 91, 207901 (2003).

[2] T. Shi, Ying Li, Z. Song, and C. P. Sun, Phys. Rev. A 71, 032309 (2005).

[3] G. Casati, B. V. Chirikov, F. M. Izraelev, and J. Ford, Lect. Notes Phys. 93, 334 (1979).

[4] S. Fishman, D. R. Grempel, and R. E. Prange, Phys. Rev. Lett. 49, 509 (1982).

[5] M. B. d'Arcy, R.M Godun, M. K. Oberthaler, D Cassettari, and G. S Summy, Phys. Rev. Lett. 87, 074102 (2001); S. Fishman, I. Guarneri, and L. Rebuzzini, Phys. Rev. Lett. 89, 084101 (2002).

[6] F. L. Moore, J. C. Robinson, C. F. Bharucha, Bala Sundaram, and M. G. Raizen, Phys. Rev. Lett. 75, 4598 (1995).

[7] B. Georgeot and D. L. Shepelyansky, Phys. Rev. E 62, 3504 (2000); Phys. Rev. Lett. 86, 2890 (2001).

[8] L. F. Santos, G. Rigolin, and C. O. Escobar, Phys. Rev. A 69, 042304 (2004).

[9] P. A. Miller and S. Sarkar, Phys. Rev. E 60, 1542 (1999).

[10] A Lakshminarayan and V Subrahmanyam, Phys. Rev. A 71, 062334 (2005).

[11] T. Prosen, Phys. Rev. E 60, 1658 (1999); 65, 036208 (2002).

[12] E. Ott, Chaos in Dynamical Systems (Cambridge University Press, Cambridge, England, 1993); L. E. Reichl, The Transition to Chaos (Springer, New York, 2004).

[13] F. C. Alcaraz, M. N. Barber, M. T. Batchelor, R. J. Baxter, and G. R. W. Quispel, J. Phys. A 20, 6397 (1987).

[14] P. H. Jones, M. M. Stocklin, G. Hur, and T. S. Monteiro, Phys. Rev. Lett. 93, 223002 (2004).

[15] A. B. Rechester and R. B. White, Phys. Rev. Lett. 44, 1586 (1980).

[16] D. A. Meyer and N. R. Wallach, J. Math. Phys. (N.Y.) 43, 4273 (2002); G. K. Brennen, Quantum Inf. Comput. 3, 619 (2003).

[17] W. K. Wootters, Phys. Rev. Lett. 80, 2245 (1998).

[18] B. G. Klappauf, W.H. Oskay, D. A. Steck, and M. G. Raizen, Phys. Rev. Lett. 81, 4044 (1998).

[19] T. J. Osborne and N. Linden, Phys. Rev. A 69, 052315 (2004). 